\title{
RENOVACIÓN HISTORIOGRÁFICA EN EL URUGUAY DE LA DICTADURA Y LA REINSTITUCIONALIZACIÓN DEMOCRÁTICA (1973-1995)
}

POR

\author{
CARLOS ZUBILLAGA
}

Universidad de la República (Uruguay)

Se ofrece un panorama de la situación historiográfica en Uruguay desde los prolegómenos del golpe de Estado (junio de 1973) hasta la actualidad, advirtiendo la renovación sistemática operada en este campo del conocimiento. La indagatoria supone develar los cambios registrados en los criterios teóricos, los intereses temáticos, las modalidades de articulación del colectivo de historiadores, la difusibilidad de la producción historiográfica y la profesionalización de la disciplina.

1. LA PRODUCCIÓN HISTORIOGRÁFICA EN LA ANTESALA DEL GOLPE DE ESTADO

A un cuarto de siglo del inicio de los estudios universitarios en Historia $^{1}$, el panorama en las vísperas de la ruptura institucional permitía apreciar un avance significativo de los criterios teóricos y las pautas metodológicas de lo que dio en llamarse la Nueva Historia.

Vaciada principalmente en el molde de la renovación historiográfica iniciada por el grupo de Annales, desarrollada al calor de las enseñanzas (o del acicate) de José Luis Romero, Tulio Halperin Donghi y Gustavo Beyhaut, la Nueva Historia tuvo en el Uruguay de la crisis sus espacios de crecimiento privilegiados (la Facultad de

1 Los cursos correspondientes al primer Plan de Estudios de la Licenciatura en Historia, de la Facultad de Humanidades y Ciencias (Universidad de la República), comenzaron a dictarse en 1948. 
Humanidades y Ciencias, el Instituto de Profesores "Artigas")2, sus articulaciones en equipos relativamente homogéneos ${ }^{3}$, sus cauces editoriales ${ }^{4}$ y críticos $^{5}$. Su principal dinamizador, en términos de una labor de largo aliento tendiente a la ampliación de la base humana de cultivo de la disciplina, fue Juan Antonio Oddone, a través de la tarea de formación de jóvenes investigadores cumplida desde la $\mathrm{Fa}$ cultad de Humanidades y Ciencias, en los Seminarios de Historia de la Cultura y de Historia del Uruguay que dirigiera entre los años 1964 y $1973^{6}$. Ese emprendimiento no dejó de expresar un modo de concebir la misión de la Universidad y del intelectual en un contexto de crisis: "El oficio de historiador — afirmaba por entonces Oddone- parece ya inseparable de una doble servidumbre que se mueve entre la exigencia técnica y el compromiso humano. Para quienes

2 Entre 1957 (año en el que regresaron las primeras promociones de Licenciados de la Facultad y de Profesores del Instituto) y 1963, ambas instituciones aportaron respectivamente 5 y 4 investigadores en Historia al proceso de renovación que venimos aludiendo.

3 Se reconocían como tales los pertenecientes al Grupo "Historia y Presente": Blanca París de Oddone, José Pedro Barrán, Luis Carlos Benvenuto, Roque Faraone, Julio Millot, Benjamín Nahum, Juan Antonio Oddone, Lucía Sala de Touron, Julio C. Rodríguez, Nelson de la Torre. Los últimos tres, a su vez, encabezaban el equipo de investigación de cuño marxista (grupo "Praxis") al que también pertenecían Rosa Alonso, Selva López, María del Carmen de Sierra, Silvia Rodríguez Villamil, Raúl Jacob y Roberto Aguirre.

4 Ediciones de la Banda Oriental, Editores Reunidos y Editorial Arca, Departamento de Ediciones de la Universidad de la República. Para el grupo "Praxis", en particular, Ediciones Pueblos Unidos.

5 Básicamente la sección de crítica bibliográfica del semanario "Marcha".

6 Casi como un "manifiesto" de la Nueva Historia, Oddone aludía a los rasgos fundamentales de la renovación historiográfica, al señalar las principales características de cinco trabajos que presentaba - emanados de la actividad curricular en la Facultad de Humanidades y Ciencias - en un volumen colectivo editado en 1968 (MOURAT - MARIANI JACOB - PELlEGRINO - Di SEGNI - RODRIGUEZ VILlAMIL, Cinco perspectivas históricas del Uruguay moderno. Montevideo, Fundación de Cultura Universitaria, 1968): "Los trabajos que fragmentariamente se reunen en este volumen intentan contribuir [...] al análisis histórico de algunos aspectos del proceso de europeización que modifica las bases económicosociales del Uruguay durante la segunda mitad del siglo pasado y los comienzos del presente. Los une, por lo pronto, la coincidencia en un área temática cada vez más urgida por el reclamo de un autoconocimiento nacional; pero también reflejan en alguna medida la influencia de una modalidad historiográfica que viene abriéndose camino dentro y fuera de los medios académicos del país. Esta corriente, que en sus inclinaciones más notorias se orienta hacia la investigación de los elementos estructurales de nuestro pasado, no desdeña el rigor documental ni la erudición bien entendida, sino que por el contrario extrae sus puntos de vista de una masa documental examinada a la luz de técnicas renovadas, retomando y reelaborando bajo nuevas y más vivas interrogaciones los resultados alcanzados por la llamada historiografía tradicional".

R. I., 1997, n. $^{\circ} 210$ 
creemos que la historia es también, y sobre todo, ciencia del presente, en momentos tan inciertos para la Universidad y para el país resulta hoy reconfortante asistir a la formación de un equipo de jóvenes que se vuelven sobre su pasado e intentan replantearse, desde su actual circunstancia, las raíces históricas de una época reciente sobre cuyas grandes contradicciones se asientan las bases del Uruguay contemporáneo"?.

La Nueva Historia hegemonizó las interpretaciones sobre el pasado del país, en convergencia con una exacerbada inquietud interrogativa que la sociedad uruguaya comenzó a practicar al avanzar la crisis socio-económica e institucional de fines de los '60. La angustia generalizada ante la situación, la enconada controversia ideológica que pautaba el momento, la ruptura de algunos de los supuestos operativos tradicionales de la política uruguaya, resultaron otros tantos motivos de inquisición hacia el pasado, en busca de una causalidad que permitiera comprender el presente y vislumbrar el futuro, cuando no exorcizar "culpas" colectivas o sectoriales. De allí que el ritmo de demanda de respuestas, proveniente del campo político-social, terminó por no encontrar satisfacción en la producción historiográfica. Las exigencias metodológicas de ésta resultaban inasimilables para un público lector cuya avidez se acompasaba al ritmo febril de los enconos políticos e ideológicos. Por lo mismo, sin dejar de operar como una referencia firmemente asentada en la respetabilidad de su origen académico, la producción historiográfica de los cultores de la Nueva Historia cedió, en buena medida, el terreno a elaboraciones menos debidas a la investigación y más a la reinterpretación, al tiempo que proclives a despejar los campos ideológicos del pasado, aun a riesgo de frecuentes anacronismos ${ }^{8}$.

Lo que entre autores militantemente comprometidos con una visión alternativa de la sociedad (como eran los del grupo "Praxis"), constituía un acicate investigador, a veces servido por un discurso

7 Juan A. Oddone, Presentación, en: Mourat [6], [p. vi].

8 Un ejemplo de este tipo de producción lo ofreció la obra de Carlos MACHADO, Historia de los Orientales (Montevideo, Ediciones de la Banda Oriental, 1972), cuyas palabras iniciales no dejaban duda al respecto: "Vamos a recorrer la historia "nacional" [...] con un par de objetivos: indagar la injerencia del imperialismo y detenernos en aquellos puntos más controvertidos y tergiversados". 
apasionado 9 , pero nunca divorciado de las exigencias científicas que a esa altura eran ya connaturales a la elaboración de conocimiento histórico, en cierto ensayismo revisionista de izquierda alcanzó carácter sustitutivo del control metodológico impuesto a la búsqueda de la verdad ${ }^{10}$. En este sentido, no hubo demasiadas diferencias con otras modalidades revisionistas (de signo ideológico bien diferente, por cierto) que habían incursionado con anterioridad en el país. Para aquellas y para estas resultaría válida la apreciación de Halperin Donghi, referida al caso argentino: "El revisionismo era, desde su origen, antes que una escuela de investigación histórica, un esfuerzo por sustituir a una cierta imagen del pasado nacional otrora juzgada más apta para justificar ciertas actitudes frente al presente. Ello suponía, sin duda, una concepción de la historia misma en la que la utilidad práctica y actual de ésta, tenía primacía sobre su dimensión propiamente cognoscitiva"11.

A pesar de la aceptación de la síntesis presentelpasado como elemento sustancial de la indagación histórica, que se enmarcaba en una realidad exigente de compromisos (para la que el divorcio entre posicionamiento socio-político y ejercicio profesional no era generalmente de recibo), la generación que hacia fines de los '60 accedía

9 Sobre este particular adviértase el sagaz comentario de Eugenio Petit Muñoz en la Advertencia que escribió para el volumen fundamental de la serie que produjeron de la Torre, Rodríguez y Sala de Tourón: "Los autores de este libro toman partido apasionadamente en favor de "los más infelices" [...] seguros de sí mismos en la justicia de su posición, adjetivan y señalan a menudo con virulencia a los elementos del bando contrario [...]. Y lo hacen con incisivo y mordiente lenguaje, que no han cuidado de depurar porque han preferido sin duda dejarlo intacto como prueba de la espontaneidad con que nació [...]. Sean perdonados, pues, por quienes no prefieran llamarlos bienvenidos, estos arrebatos que tan crudamente concurren con los juicios expresados en el tono de una admonición más serena pero no menos justa, para poner al desnudo unas formas tan siniestras de la verdad histórica" (Nelson DE LA TORRE/ Julio C. RODRIGUEZ).

Lucía SALA de TOURON, La revolución agraria artiguista (1815-1816), Montevideo, Ediciones Pueblos Unidos, 1969, pp. 10/11.

10 El caso del ensayo de Julio A. Louis, Batlle y Ordóñez. Apogeo y muerte de la democracia burguesa (2a. edición. Montevideo, Nativa Libros, 1972) resultó paradigmático. La justificación del mismo que el autor brindaba en las Notas a la 2a. Edición, exime de todo comentario: "Pienso que aunque mínimamente, puede ayudar a la gran tarea histórica del proletariado. Tarea que requiere, hoy por hoy, la creación de un legítimo partido de la clase, para lo cual, el proletariado tendrá que derrumbar los falsos edificios levantados por el liberalismo y el revisionismo burgueses. Uno de cuyos pilares sostenedores que más dificulta su misión es la adulteración histórica del país en que lucha".

11 Tulio HalPERIN DONGHI, El revisionismo histórico argentino. Buenos Aires, 1970, p. 25.

R. I., $1997, \mathrm{n}^{\circ} 210$ 
al campo historiográfico, no dejaba de precaverse del riesgo de enajenación del paradigma científico. En un sugerente trabajo sobre la Historia y los jóvenes ${ }^{12}$, Silvia Rodríguez Villamil esbozaba algo así como una declaración de principios: "[...] en el nuestro, como en otros países, la nueva orientación del análisis histórico no obedece a motivaciones exclusivamente teóricas y racionales; sino a una toma de conciencia por parte de los jóvenes del estado en que viven nuestros pueblos [...] Y en este punto aparece un rasgo característico de los jóvenes como historiadores: si bien hay un deseo de incidir sobre la realidad, y se tiene conciencia de que todo análisis histórico parte de una perspectiva ideológica forjada en el presente, se pone un especial cuidado en no deformar los hechos según nuestra voluntad. Hay una marcada preocupación por conocer directamente la realidad; para nosotros el objetivo primordial de la Historia es el conocimiento, es hacer inteligible nuestro pasado, y no forzarlo para lograr la demostración de alguna tesis que a priori consideremos positiva"13.

Al tiempo que la Nueva Historia priorizó los temas relacionados con las estructuras sociales y económicas, y postergó la Historia Política (que había enseñoreado por décadas la producción historiográfica tradicional), aceptó el desafío del diálogo interdisciplinario. Comenzó, aunque tímidamente, una apertura hacia la Economía, la Sociología y la Ciencia Política, en la que no siempre se tuvo la certidumbre del propio valer en cuanto instrumento cognoscitivo, lo que sesgó algunos aportes ${ }^{14}$. Sin perjuicio de ello, otras propuestas apuntaron a la fertilidad de un relacionamiento (el de la Historia y la Ciencia Política) que recién adquiriría estatuto propio hacia fines del período dictatorial; ejemplo de ello lo ofrece, en vísperas de la ruptura institucional, el trabajo de Carlos Real de Azúa Partidos, políti-

12 Silvia Rodriguez Villamil, "Nuestra Historia y los jóvenes", en: GalEano RUFFINELLI - RODRIGUEZ VILlAMIL, "El mensaje de los jóvenes", en Enciclopedia Uruguaya. 57. Montevideo, Diciembre de 1969.

13 Ibidem, pp. 137/138.

14 Un ejemplo de este ambiguo relacionamiento lo ofrecen los "Enfoques sobre el período batllista" contenidos en Cuadernos de Ciencias Sociales. 2. Montevideo, Facultad de Derecho y Ciencias Sociales - Instituto de Ciencias Sociales, 1972. 
ca y poder en el Uruguay (1971 - Coyuntura y pronóstico $)^{15}$, quizás el producto pionero de la neo-historiografía política uruguaya ${ }^{16}$.

La difusibilidad del conocimiento histórico ${ }^{17}$, su funcionalidad ideológico/política, la permeabilidad de la sociedad uruguaya ante una reflexión diacrónica de la realidad ${ }^{18}$, constituyeron elementos concurrentes a la "satanización" de la Nueva Historia una vez producido el golpe de Estado de junio de 1973 y, sobre todo, la intervención de la Universidad de la República en octubre del mismo año. Comenzarían a partir de entonces las múltiples modalidades de la dispersión de los equipos de investigadores: exclusión de los ámbitos públicos (ceses infundados, sumarios que culminaban en des-

15 Aparecido en Uruguay hoy (Buenos Aires, Siglo Veintiuno, 1973) y reeditado en 1988 por la Facultad de Humanidades y Ciencias.

16 En el Prólogo que para la reedición de este texto escribí en 1988 señalé al respecto de la fecundidad interdisciplinaria de la obra de Real de Azúa: "La estructura de este ensayo denota dos vertientes disciplinarias privilegiadas por su autor en cuanto cauces de conocimiento, a la vez que trasunta su preocupación por servir la reflexión mediante una apuesta consciente a la excelencia comunicativa. La Ciencia Política - en agraz entre nosotros al despuntar la década pasada [me refiero a la de los '70] - y la Historia -morosamente transitada por Real de Azúa a lo largo de toda su producción - confluyen en el esfuerzo cognoscitivo. El texto del ensayo apela fundamentalmente a los aportes de la primera [...], en tanto que el arsenal de Notas (una cuarta parte aproximadamente del espacio global del ensayo) denota los aportes de la Historia. División de modos cognoscitivos que ni es tajante (hay valiosas reflexiones históricas en el texto y no pocas "pistas" politológicas en las Notas), ni supone una suerte de servidumbre (o auxilio) de la Historia respecto de la Ciencia Política, pero que evidencia si el carácter fuertemente historizante que Real de Azúa concebía como inexcusable a la hora de abordar el campo politológico (en una advertencia que, dicho sea de paso, no siempre tuvieron presente quienes transitaron en nuestro país los senderos de la Ciencia Política)" (ob. y edic. cit., pp. 7/8).

17 Dieron hacia fines de los '60 un impulso fundamental a la difusión del conocimiento histórico, dos series fasciculares publicadas casi coetáneamente y que circularon en tirajes de muchos miles de ejemplares: Capítulo Oriental y Enciclopedia Uruguaya. La primera, debida a un emprendimiento del Centro Editor de América Latina, constituyó una Historia de la literatura uruguaya que se estructuró en el cauce de la Historia intelectual. Carlos Real de Azúa elaboró o revisó la mayor parte de los fascículos. Por su parte, Enciclopedia Uruguaya fue el resultado de una convergencia editorial (Editores Reunidos y Editorial Arca), sobre la base de un plan general de Angel Rama. El director ejecutivo (Luis C. Benvenuto) y el asesor historiográfico (Julio C. Rodríguez) fueron hombres del grupo "Historia y Presente".

18 Sobre este aspecto resulta de ineludible consulta el trabajo de dos jóvenes investigadoras, originado en un Seminario de Historia de la Historiografía en la Facultad de $\mathrm{Hu}$ manidades y Ciencias de la Educación: Isabela Cosse - Vania MARKARIAN, Memorias de la Historia. Una aproximación al estudio de la conciencia histórica nacional. Montevideo, Ediciones Trilce, 1994.

\section{R. I., $1997, \mathrm{n}^{\circ} 210$}


tituciones, renuncias forzadas), exilio o emigración, reclusión dispersa en nuevos espacios institucionalizados de la esfera privada.

\section{LA RECONSTRUCCIÓN DEL ESPACIO HISTORIOGRÁFICO}

La inmediata consecuencia de los sucesos políticos de 1973 en referencia al desarrollo historiográfico se manifestó, como se ha señalado, en el bloqueo de la continuidad de la labor de los investigadores nucleados en colectivos de matriz ideológica o en las estructuras de la enseñanza superior. Al mismo tiempo se reasignó a la Historia un papel convalidador de opciones ideológicas (en este caso, destinadas al sustento del "proceso" militar), en tanto el pasado era apreciado como el reservorio de los valores "puestos en peligro" por la crisis política de los años precedentes. Tal utilización de la Historia se hizo evidente en la celebración del denominado "Año de la Orientalidad"19, punto de inflexión del intento de retrotraer la "unidad del proceso histórico nacional" al momento previo a la emergencia de los partidos, en consonancia con la propuesta neocorporativista impulsada por el Presidente Bordaberry. Superado este proyecto, la acción gubernamental en el campo historiográfico se orientó a reformular los planes de estudio de los ámbitos de formación superior. En 1977 se impusieron nuevos planes en la Licenciatura de Historia (de la Facultad de Humanidades y Ciencias) ${ }^{20}$ y en

19 Se adjudicó tal denominación al año 1975, en el que se cumplió el sesquicentenario del proceso independentista de 1825. La apelación al concepto de "orientalidad" denotó no sólo el anacronismo de sus promotores - que saltaban de la configuración criolla a la modernidad sin solución de continuidad - sino la aviesa intención de descalificar al opositor a la dictadura mediante la negación de su rasgo de identidad.

20 El Plan de Estudios 1977 suprimió asignaturas historiológicas (Teoría y Metodología de la Historia, Filosofía de la historia), enfatizó la temática de la Historia militar, prohijó una concepción basada en la influencia preponderante del individuo en el proceso histórico (apelando a un enfoque biográfico de cuño psicologista), y erradicó los instrumentos esenciales de la formación de investigadores. La incorporación de tres asignaturas denominadas Pedagogía I, II y III, pretendió reorientar la formación de Licenciados hacia una eventual incorporación de los mismos en el ámbito de la enseñanza formal, no tanto atendiendo a una demanda sobre el perfil ocupacional de aquéllos, que reconocía antecedentes en la prédica de los egresados universitarios, sino más bien a una posibilidad de incidir en la Enseñanza Media con recursos humanos formados en la nueva orientación de la disciplina. 
el Profesorado en Historia (del Instituto de Profesores “Artigas") ${ }^{21}$ con la finalidad de reconvertir la disciplina en un ejercicio fundamentalmente fáctico y escasamente interpretativo, en consonancia con el papel que se estimaba propio de la enseñanza en humanidades (ajeno a la promoción de cuestionamientos sobre el objeto de estudio formulados desde las preocupaciones del presente).

Los historiadores que permanecieron en el país, aunque alejados forzosamente de los ámbitos de actividad pública, pertenecían a las dos primeras generaciones de la Nueva Historia y habían desarrollado su labor al margen del espacio privado, salvo en la relación con las editoriales que canalizaban la producción historiográfica, a las que tampoco podía identificarse como expresiones típicas de este espacio. Si bien los procesos personales de reinserción laboral (alternativa a la tradicional de la enseñanza pública, con su correlato de tiempo de investigación) resultaron variados, por lo general estos historiadores fueron confluyendo hacia una experiencia inédita para el "modelo" de trabajo académico uruguayo: la de incorporación a instituciones privadas de investigación (organizaciones no gubernamentales, autogestionadas, integradas por investigadores en ciencias sociales provenientes - casi en su totalidad - del ámbito universitario) ${ }^{22}$.

Estos centros privados de investigación constituyeron una respuesta del mundo académico, a la exclusión en la Universidad intervenida tanto del principio de libertad de cátedra, como del cultivo en base a criterios de rigurosidad y excelencia - de las ciencias sociales, en tanto instrumentos para la comprensión de la realidad y su transformación. La clave de funcionamiento de estos centros radicó en el acceso al financiamiento externo, lo que impuso ritmos y modalidades de validación de los resultados que por lo general habían sido desconocidos hasta entonces en el ambiente universitario (mucho más parsimonioso en el manejo del tiempo investigativo y

21 Se redujo el tiempo de formación curricular de cuatro a tres años, eliminándose también las asignaturas que conferían sustento teórico-metodológico a la disciplina.

22 En un trabajo dedicado al tema a escala del Cono Sur, Brunner y Barrios han denominado a estas instituciones Centros Académicos Independientes (CAI), señalando: "Los CAI uruguayos asumen, desde su propia conformación, la doble responsabilidad de continuar en el país la tradición de las disciplinas excluídas de la Universidad, o recortadas dentro de ella en función de intereses ideológicos precisos, y de mantener vivo el análisis crítico de la realidad nacional" (José Joaquín BRUNNER - Alicia BARRIos, Inquisición, mercado y filantropía. Ciencias Sociales y autoritarismo en Argentina, Brasil, Chile y Uruguay. Santiago de Chile, FLACSO, 1987, p. 142).

R. I., $1997, \mathrm{n}^{\circ} 210$ 
menos acostumbrado a soportar instancias de competitividad). Un rasgo común a la mayoría de estos centros estribó en el cultivo de varias disciplinas - aunque con énfasis o rangos de atención diferenciados ${ }^{23}$ - , circunstancia que contribuyó a un diálogo interdisciplinario que, con anterioridad, apenas había comenzado entre historiadores y economistas, aunque con desconfianzas no superadas.

Si bien la labor sustantiva de estos centros -y aquella que les permitía obtener recursos por la vía de proyectos o subvencionesradicaba en la investigación, su propia existencia como ámbitos plurales en los que se cultivaba un conocimiento que resultaba alternativo al que sustentaban los titulares y beneficiarios del poder, les confirió el perfil de "islas de libertad" en un contexto autoritario y represivo. Que esas "islas" resultaran vagamente "amparadas" por su pertenencia a redes regionales de ciencias sociales (CLACSO, PISPAL), por su relacionamiento con instituciones financiadoras de la investigación (Fundación Ford, Social Science Research Council, IDRC, Fundación Guggenheim, SAREC) o por su vinculación con organismos internacionales (UNESCO, CEPAL, OIT, SELA), las tornó referencias inexcusables para las nuevas generaciones, constreñidas fuertemente en el ámbito universitario. De allí que en algunos de esos centros se sumara a la actividad de investigación, la de enseñanza en núcleos reducidos o la de formación de recursos humanos mediante mecanismos de pasantía o de ayudantía.

En el campo de la formación de recursos el CLAEH implementó un plan más ambicioso, iniciando en 1977 sendos Seminarios de Formación de Investigadores en Historia, Sociología ${ }^{24}$ y Econo-

${ }^{23}$ El Centro de Investigaciones Económicas (CINVE), aunque alojó a historiadores (José Pedro Barrán y Benjamín Nahum estuvieron de algún modo adscritos a sus programas, si bien a través de una modalidad relativamente autónoma), priorizó las investigaciones en Economía. De modo semejante, el Centro de Investigaciones y Estudios- Uruguay (CIESU), que enfatizó el trabajo en Sociología, dio acogida a Juan Rial, Jaime Klaczko y Angel Cocchi, quienes bajo la orientación inicial de Carlos Real de Azúa abordaron un programa de investigación en Historia Urbana y Regional. El Centro Interdisciplinario de Estudios sobre el Desarrollo (CIEDUR) tuvo una línea de investigación en Historia a cuyo frente operó Raúl Jacob. El Centro Latinoamericano de Economía Humana (CLAEH) constituyó a partir de 1979 un Programa de Investigaciones en Historia bajo la orientación de Carlos Zubillaga. CIEDUR y CLAEH fueron los dos centros más nítidamente interdisciplinarios, en tanto conjugaron programas en Economía, Sociología, Historia y Ciencia Política, aunque no siempre todos ellos felizmente interrelacionados.

24 Estuvo a cargo de Horacio Martorelli. 
mía ${ }^{25}$. El Seminario de Historia, estructurado curricularmente en tres años, sobre un esquema de tratamiento de temas teóricos, metodológicos y técnicos, con labor de investigación guiada que variaba temáticamente año a año, estuvo a cargo de Carlos Zubillaga y tuvo tres ediciones ${ }^{26}$. Constituído en el ámbito privilegiado de reclutamiento del Programa de Historia del CLAEH, el Seminario de Formación de Investigadores se convirtió en la única experiencia sistemática de capacitación especializada que se desarrolló durante los años de la dictadura en el país.

Los centros privados de investigación fueron, asimismo, espacios de convocatoria para la realización de las primeras reuniones académicas regionales sobre temas históricos que se cumplieron en el país luego del golpe de Estado. Ello modificó las prácticas previas al '73, más articuladas en la convergencia de visiones sobre el pasado y en la significación "pragmática" del conocimiento histórico, que en la contrastación de perspectivas interpretativas ${ }^{27}$.

En el lapso comprendido entre $1975^{28}$ y 1984 los centros privados de investigación permitieron revitalizar el conocimiento histórico, no sólo mediante la recuperación de anteriores enfoques sobre algunos temas, sino por el abordaje de nuevas líneas de indagación,

\begin{abstract}
25 Estuvo a cargo, primeramente, de Alberto Tisnés y, posteriormente, de Walter Cancela.

26 Cumplidos entre 1977 y 1985 estos Seminarios contaron en total (en sus tres ediciones) con veinte estudiantes. Formaron parte de la primera generación Gerardo Caetano, Ana Frega, Jorge Balbis, Yvette Trochón, Mónica Maronna y Rosita Pellerey. Al evaluar a fines de 1979 la experiencia cumplida, uno de los participantes del primer Seminario señalaba, enfatizando su apreciación sobre la modalidad de trabajo elegida: "Dentro de un contexto francamente adverso a las realizaciones comunitarias, donde la formación intelectual es encarada con un egoísta criterio de individualismo, el haber tenido la oportunidad de compartir el esfuerzo y las búsquedas de estos años con un grupo humano de singulares características [...] se perfila como uno de los aspectos más destacables del seminario".

27 El CLAEH organizó, con estas características, y siempre mediando el apoyo institucional del Consejo Latinoamericano de Ciencias Sociales (CLACSO), tres encuentros entre historiadores de la región: en 1982 el Seminario sobre "Modernización y sistema político en el Río de la Plata (1875-1933)"; en 1986 el I Seminario sobre Historia del Movimiento Sindical en América Latina; y en 1989 las Jornadas Rioplatenses de Historia Comparada sobre "Respuestas reformistas al Estado oligárquico, 1890-1930". En estos eventos llegaron a participar historiadores argentinos, paraguayos, chilenos, ecuatorianos, portorriqueños, costarricenses, mexicanos y uruguayos.

28 En ese año se fundaron CIESU y CINVE, mientras CLAEH (que estaba constituído desde 1957, aunque con un perfil acotado a la Sociología Rural, en el marco del pensamiento lebretiano) se encontraba en los primeros pasos de su recomposición académica.
\end{abstract}


así como por el tránsito (y revaloración) de fuentes no tradicionales. La producción historiográfica de ese período - que coincide prácticamente con el de la dictadura - asumió sutilmente un papel de contestación al régimen, logrando un difícil equilibrio entre sus posibilidades como narración y como interpretación del pasado. Sobre todo en relación con interrogantes que la sociedad se formulaba angustiosamente para entender las raíces de la crisis que padecía y, sin duda, avizorar los cauces de salida ${ }^{29}$.

La singular experiencia de los centros privados de investigación no se agotó al producirse el tránsito hacia la reinstitucionalización del país; más bien adquirió en esos tiempos (desde 1983 hasta 1987) un perfil más acusadamente incisivo en la interpretación de la realidad, y en la formulación propositiva. La recuperación a partir de 1985 de los espacios tradicionales de producción del conocimiento histórico (los universitarios), no hizo variar de inmediato la posición de los centros privados de investigación, por más que quienes actuaban en el seno de éstos tuvieron un papel preponderante en la reinstalación de aquéllos; pero el cúmulo de acciones que la recuperación de la autonomía universitaria, la configuración de nuevos cuadros docentes y la revisión de los planes de estudio impuso a numerosos investigadores, hizo que éstos continuaran desarrollando en los centros privados buena parte de su tarea de generación de conocimiento. Hacia fines de la década de los '80 tanto las restricciones en el financiamiento externo, como la demanda de no pocos de sus cuadros para el cumplimiento de funciones políticas o de dirección universitaria, afectaron a los centros privados haciéndoles perder su gravitación insoslayable durante más de una década en la producción de conocimiento. Es de advertir, asimismo, que el tránsito de numerosos investigadores universitarios por la experiencia de los centros

29 En una lúcida evaluación de la historiografía del período, José Pedro Barrán afirmaba al iniciarse la reinstitucionalización del país: "[...] no puede negarse que la dictadura coincidió con una singular creatividad en las ciencias sociales, particularmente en Economía e Historia. [...] investigadores de izquierda, cesantes en la Universidad, fundaron o remozaron centros privados, que florecieron como hongos, y así ciertas siglas pasaron a ser conocidas como refugios de la inteligencia: CLAEH, CINVE, CIESU, CIEDUR, etc. [...] la dictadura generó la curiosidad intelectual, el mayor desafío interpretativo de un siglo XX que la mayoría de los uruguayos nunca imaginaron llegara a esos extremos y que veían insuficientemente explicados por sus historiadores clásicos" (José Pedro BARRAN, "Dictadura e Historia. El 'boom' historiográfico", en: Brecha. Año I, Nº. Montevideo, 15 de noviembre de 1985). 
privados, generó al retornar aquellos a la Universidad, el trasvasamiento de prácticas que produjeron una fuerte revisión de los criterios operativos hegemónicos en el espacio universitario con anterioridad al '73, en especial los referidos a la evaluación de la producción científica, a la competencia por la obtención de recursos, a los tiempos investigativos, a la participación en redes científicas internacionales, etc.

\section{LOS CAMBIOS TEÓRICOS Y LA DIVERSIFICACIÓN DIFUSORA}

Apartada de las urgencias ideológicas que caracterizaran buena parte de la producción anterior al '73, aunque demandada por urgencias académicas no menos severas, la historiografía uruguaya del período dictatorial apeló más a una racionalización de la comprensión del pasado que habilitara cierta prognosis, que a la convalidación empírica de un supuesto teórico concebido como el idóneo para reivindicar caminos y deslindar responsabilidades. En este sentido resultó menos tributaria del esfuerzo de la Nueva Historia de los '60 y primeros '70, en lo que constituyera la clave de ésta: la pertinencia de una desagregación del pasado que privilegiara el análisis de las estructuras sociales y económicas. La preocupación por las técnicas de cuantificación (que había constituído uno de los reclamos estudiantiles para la reforma del plan de estudios 1960 de la Licenciatura de Historia en Humanidades) se debilitó, frente a la revalorización de tipos de fuentes hasta entonces escasamente transitadas y que requerían un aguzamiento de las técnicas cualitativas. Asimismo, se advirtió la necesidad de auscultar con nuevas preocupaciones indagatorias los archivos nacionales (públicos y privados), a partir del reconocimiento de que en los mismos seguían durmiendo series documentales con virtualidad hermenéutica similar - si no superior-a la que ofrecían los repositorios europeos, cuya consulta había cautivado a los historiadores de la primera generación de la Nueva Historia.

Asimismo, se concibió la Historia como una disciplina requerida por un frecuente diálogo con las restantes ciencias sociales, en términos de interactuación en el campo teórico-metodológico, tanto como en el de conjugación de esfuerzos por abordar de manera más integral los fenómenos sociales. Al mayor rigor teórico de otras disciplinas (como la Ciencia Política o la Sociología), frecuentemente

R. I., 1997, n. 210 
afectadas por un sincronismo esterilizante o bloqueadas por la indefinición epistemológica, la Historia respondió con su viejo(/renovado) apego a los hechos como sustento ineludible de una interpretación fecunda y su valorización del tiempo como factor sin el cual es imposible aprehender la realidad social. La interlocución no resultó fácil, aunque dejó huellas que reaparecieron varios años más tarde -entre 1986 y 1990 - cuando en el seno de la Universidad de la República se discutió la reestructura del Area Académica Social y la adscripción institucional de diversas ciencias ${ }^{30}$.

La historiografía uruguaya en el período dictatorial dio cuenta, además, de una renovación teórica, escasamente explicitada, pero notoria en la abundante producción édita: la relacionada con un esfuerzo de comprensión del pasado que apostó a la desagregación de los espacios sociales, para indagarlos en profundidad a partir de especializaciones hasta el momento no desarrolladas en el país, con la finalidad de recomponer el todo sobre nuevos esquemas de articulación. La caducidad de los abordajes que daban por resuelto (previa e ineludiblemente) el marco teórico de la investigación, resultó un componente de la producción historiográfica del período, que alcanzó así una libertad de interrogación, una disposición "revisionista" de ciertas interpretaciones consagradas, una sutileza para advertir peculiaridades y disfunciones, que la volvió menos previsible en sus resultados. De allí quizás el interés renovado que logró suscitar en el público no especializado. La difusión de las investigaciones alcanzó un grado apreciable de celeridad. A diferencia de lo que sucedió en

\footnotetext{
30 El debate que tuvo lugar en el seno de la Universidad de la República (por cierto, merecedor de un análisis más detenido del que aquí apenas se puede insinuar), culminó cuando en 1990 se creó la Facultad de Ciencias Sociales (a la que se adscribieron las Licenciaturas en Ciencia Política y en Sociología) y se reestructuraron la Facultad de Humanidades y Ciencias de la Educación (en la que permanecieron las Licenciaturas en Ciencias Históricas, en Lingüística y en Ciencias Antropológicas), la Facultad de Ciencias Económicas y de Administración (que conservó la Licenciatura en Economía) y la Facultad de Derecho (que retuvo la Licenciatura en Relaciones Internacionales), todo ello sin perjuicio de la permanencia en estas dos últimas de las formaciones profesionales típicas (Abogacía, Notariado, Diplomacia, Contabilidad, Administración). La controversia escapó al plano epistemológico para traducir desconfianzas sobre el incremento de "poder" que derivaría de la concentración en una sola estructura del conjunto de las ciencias sociales más desarrolladas.
}

R. I., $1997, \mathrm{n}^{\circ} 210$ 
los '60, el "boom" historiográfico no radicó en la línea ensayística, sino en la más rigurosa de la investigación ${ }^{31}$.

La difusión de los resultados de la investigación historiográfica no sólo se canalizó en las ediciones de libros (las Editoriales Arca y Banda Oriental fueron los principales vehículos de esta labor), sino en la aparición de numerosas publicaciones mimeografiadas (presentadas con extrema modestia gráfica: apenas unas tapas de cartulina, generalmente con un troquelado para la visualización del título y el autor) que fueron el instrumento de los centros privados, para dar a conocer rápidamente los avances o informes finales de las investigaciones que se desarrollaban en su seno (lo que era, por otra parte, un cuasi-requisito de los financiamientos externos). Hacia el final del período dictatorial, la política de difusión de la investigación en ciencias sociales implementada por los centros intentó canalizarse mediante la reiteración de la exitosa experiencia de las series fasciculares de fines de los ' $60^{32}$, logrando una respuesta relativa; ello parecería indicar que el público lector se había acostumbrado, a esa altura, a otros tipos de abordajes ${ }^{33}$.

Una novedad en la difusibilidad del conocimiento histórico estuvo dada durante el período dictatorial por la emergencia de una revista especializada en ciencias sociales (los Cuadernos del CLAEH)

\footnotetext{
31 Cerca de cuarenta títulos, entre libros y ediciones mimeografiadas (avances de investigaciones o informes finales de las mismas) se publicaron entre 1975 y 1984.

32 En 1983 el CLAEH editó una serie de diez fascículos bajo el título El Uruguay de nuestro tiempo. El referido a Los partidos políticos, escrito por Carlos ZUBILLAGA y Romeo PÉREZ, fue el fruto de una conjunción de análisis desde los campos de la Historia y la Ciencia Política. Blanca PARÍs DE ODDONE prologando la edición encuadernada de los fascículos, apuntaba a la significación historiográfica que adquiría la serie más allá de la especificidad disciplinaria de sus abordajes temáticos: "Los trabajos [...] constituyen sin duda un aporte primicial en el conocimiento de nuestro pasado más reciente. Pero asimismo invitan a reflexionar sobre los cambios que se vienen procesando en el mundo actual y especialmente en el área latinoamericana, contexto hacia donde el Uruguay habrá de mirar insistentemente en los próximos años cuando el país entero asuma la tarea nacional de reconstrucción a la que todos nos debemos [...]".

33 Cuando en 1989 Ediciones de la Banda Oriental realiza un intento similar, bajo el título de El Uruguay de la dictadura, 1973-1985, confiriendo "la responsabilidad intelectual de [la] realización [...] en buena parte, a diversos centros e institutos de investigación de nuestro país" (CIEDUR, CIESU, CINVE, CIEP, CLAEH, CEU-FHC, Instituto de Economía), la experiencia fracasa en los primeros números. Alcanzan a publicarse dos fascículos de contenido histórico, que traducen el diálogo entre Historia y Ciencia Política: Carlos Zubillaga-Romeo Perez, La democracia atacada [Fascículo 1] y Gerardo CAETANO-José Rilla, La era militar [Fascículo 2].
} 
que se editó ininterrumpidamente desde 1976, y en cuyas páginas aparecieron numerosos artículos que daban cuenta de los avances de investigaciones en curso, en el ámbito de la propia institucion editora como fuera de ella ${ }^{34}$. Hacia el final del período una experiencia peculiar se abrió paso en el campo de la difusión del conocimiento histórico: la revista "Hoy es Historia"35. Debida al impulso de Alfonso Fernández Cabrelli, que en los '60 cultivara un revisionismo antimperialista vinculado ideológicamente con las opciones más radicales que operaban en la sociedad uruguaya y en 1984 adheriría a la estrategia de reinstitucionalización que orientaba el lider nacionalista Wilson Ferreira Aldunate, se presentó como "un medio a través del cual [tuvieran] la oportunidad de expresarse y hacer conocer el resultado de sus estudios, tanto los historiadores ya prestigiados, como aquéllos que recién se incorpora[ba]n a estas tareas con vocación y espíritu científico". Más allá de los propósitos enunciados, Hoy es Historia presentó niveles desparejos en la producción (no sólo historiográfica, sino arqueológica y antropológica) que incorporó a sus páginas, aunque constituyó un instrumento de divulgación de incidencia a escala nacional nada desdeñable.

\section{LOS CAUCES TEMÁTICOS DE LA RENOVACIÓN HISTORIOGRÁFICA}

Con origen en los cambios teóricos y metodológicos que la producción historiográfica registró en los años de la dictadura, acrecentados en su significación por el cuestionamiento de los paradigmas totalizadores en ciencias sociales, la línea de acción predominante en los '80 dio como resultado la consagración de una diversificada capacidad indagatoria que innovó en los campos de aplicación, fomentó la labor en equipo como práctica constante, incorporó aportes de otras disciplinas científicas, no se detuvo ante el criterio de autoridad (implantando un "revisionismo" de nuevo cuño, es decir, no ideológico sino científico), abrevó en fuentes nuevas u obtuvo "respuestas" nuevas de fuentes ya conocidas, acrecentó la preocupación por el discurso historiográfico y se abocó a vincular sus

\footnotetext{
34 Entre el $N^{\circ} 1$ (1976) y el No 33. (Marzo/1985) los Cuadernos del CLAEH publicaron 25 artículos sobre temas históricos, un $17 \%$ del total de los inclúídos en esos números.

35 Su No 1 apareció datado en Diciembre 1983-Enero 1984.
} 
resultados con los producidos en otros países por quienes abordaban temáticas similares (sentando las bases de una todavía incipiente Historia comparada).

A los equipos de la primera generación de la Nueva Historia (Barrán/Nahum, París/Oddone, De la Torre/Rodríguez/Sala de Tourón) sucedieron nuevas convergencias uni o interdisciplinarias (Nahum/Trochón/Maronna, Caetano/Rilla, Caetano/Jacob, Sala de Tourón/Alonso, Zubillaga/Balbis, Zubillaga/Pérez, Zubillaga/Cayota, Frega/Maronna/Trochón, Rial/Klaczko, Rodríguez Villamil/Sapriza), dando cuenta de una modalidad que aventó — quizás no todavía definitivamente- las desconfianzas o las desavenencias propias del quehacer individualista.

La Historia Política retornó al campo de las preocupaciones historiográficas como fruto de la grave crisis institucional, de la multiplicación de interrogantes que la sociedad se formuló sobre las causas del deterioro de sus tradicionales relaciones de convivencia pacífica y de la emergencia de un saber politológico moderadamente configurado. Lo que podría llamarse quizás con mayor precisión neohistoriografía política tuvo su origen en las tempranas preocupaciones registradas en los Programas de Historia y de Ciencias Políticas del CLAEH en torno a las disidencias del tradicionalismo político, a la conceptualización del batllismo y a la naturaleza y concreción del pluralismo político en Uruguay ${ }^{36}$.

La renovación de la Historia Política supuso el análisis de actores diversos de los tradicionalmente aceptados (los partidos políticos), dando entrada a la significación política de concepciones y actitudes de sujetos colectivos no partidistas, como por ejemplo la Iglesia $^{37}$ o las organizaciones representativas de intereses corporati-

\footnotetext{
36 Cfr.: Alfredo CASTEllanos - Romeo PeREZ, El pluralismo. Examen de la experiencia uruguaya (1830-1918). Montevideo, CLAEH, 1981; Ana FREGA, El pluralismo uruguayo (1910-1933). Cambios sociales y políticos. Montevideo, CLAEH, 1987; Alfredo CASTEllanos, El pluralismo uruguayo (1919-1933). El dislocamiento de los partidos. Montevideo, CLAEH, 1987; Carlos ZuBILlaGA, Las disidencias del tradicionalismo. El Radicalismo Blanco. Montevideo, Ed. Arca/CLAEH, 1979; Jorge BALBIS - Gerardo Caetano - Ana Frega - Mónica Maronna - Yvette Trochon - José Pedro Rilla - Carlos ZuBILlaGA, El primer batllismo. Cinco enfoques polémicos. Montevideo, Ed. de la Banda Oriental / CLAEH, 1986.

37 Cfr.: Romeo PEREZ, Los cristianos y la política en el Uruguay. Montevideo, Ed. Nuevo Mundo, 1986; Carlos ZuBILlaGA - Mario CAYOTA, Cristianos y cambio social en el Uruguay de la modernización, 1895-1919. Montevideo, Ed. de la Banda Oriental / CLAEH,
} 
$\operatorname{vos}^{38}$. Esta tendencia no anuló la consideración de los partidos en tanto componentes fundamentales del sistema político, si bien obligó a una revisión de su funcionalidad en el esquema democrático ${ }^{39}$; a este respecto, incluso se formularon tesis que han generado ricas controversias interpretativas, como la de la "partidocracia" uruguaya ${ }^{40}$.

Más recientemente, el desafío de la neohistoriografía política ha comenzado a transitar por el tema de la comunicación proselitista en contextos electorales, en el marco de un programa de investigación de la Facultad de Humanidades (Recuperación y sistematización de fuentes perecederas para la Historia Política del Uruguay contemporáneo), que habilita un diálogo interdisciplinario con la Antropología Política, la Semiótica y las Ciencias de la Comunicación.

La Historia Económica reafirmó su presencia en el contexto historiográfico, pero a diferencia de la producción previa al '73 (en la que se conjugaban los aportes de economistas e historiadores), con un protagonismo fuerte de los historiadores formados en la Facultad de Humanidades y en el IPA, en especial, de Raúl Jacob (que puede ser considerado el especialista más nítidamente consagrado a un espacio desagregado del conocimiento histórico) y de Benjamín Nahum.

1989; Carlos Zubillaga, "La primera Democracia Cristiana en Argentina y Uruguay", en: Cuadernos del CLAEH. 2a. Serie. Año 14. N ${ }^{\circ}$ 50. Montevideo, 1989/2.

38 Cfr.: Gerardo CAETANo, La agonía del reformismo (1916-1925). Montevideo, CLAEH, 1983; Gerardo CAETANO - Raúl JACOB, El nacimiento del terrismo. Tres tomos. Montevideo, Ediciones de la Banda Oriental, 1989-91.

39 Cfr.: Juan RIAL, Notas sobre el sistema de partidos en el Uruguay (1904-1971). Montevideo, CIESU, 1982; Relaciones interpartidarias y con actores políticos no partidarios de la oposición frente a regímenes autoritarios en Uruguay, 1933-42, 1973-83. Montevideo, CIESU, 1984; Carlos ZuBILlaGA, El dificil camino de la participación política. Población, ciudadanía y electorado (1898-1918), en: Fernando DEvoTo - Marcela FERRARI (Comp.), La construcción de las democracias rioplatenses: proyectos institucionales y prácticas políticas, 1900-1930. Buenos Aires, Ed. Biblos, 1994.

40 Algunos de los componentes de esas visiones divergentes se esbozaban en los trabajos contenidos en el volumen colectivo Gerardo CAETANo/José Pedro RiLla - Pablo Mieres - Carlos Zubillaga, De la tradición a la crisis. Pasado y presente de nuestro sistema de partidos. Montevideo, Ed. de la Banda Oriental / CLAEH, 1985. Por la formulación de la tesis de la partidocracia, cfr.: Gerardo CAETANO - José RILLA - Romeo PEREZ, "La partidocracia uruguaya. Historia y teoría de la centralidad de los partidos políticos", en: Cuadernos del CLAEH. № 44. Montevideo, 1987. Una reafirmación de esta tesis puede consultarse en: Gerardo CAETANO - José RILLA, "La partidocracia uruguaya en busca de un espejo", en: Cuadernos del CLAEH. 2a. Serie. Año 14. № 50. Montevideo, 1989/2.

R. I., $1997, \mathrm{n}^{\circ} 210$ 
La culminación de la monumental Historia Rural del Uruguay Moderno (1967-78) y la elaboración de la nueva serie Batlle, los estancieros y el Imperio Británico (Ocho tomos. Montevideo, Ediciones de la Banda Oriental, 1979-1989), ubican a José Pedro Barrán y Benjamín Nahum como los que de manera más global han contribuído al análisis del proceso socio-económico del país durante el período modernizador y hasta la crisis del sistema capitalista en 1929. Obras de excepcional envergadura, en las que predomina un exhaustivo trabajo hemerográfico, al que se adiciona la compulsa documental en archivos británicos, trascienden la Historia Económica en sentido estricto, para incursionar en aspectos sociales, políticos, demográficos y culturales; el rasgo religante aparece, sin embargo, configurado por el análisis de las relaciones de producción y sus correlatos sociales. En tanto Barrán inició posteriormente otras líneas de indagación, Nahum permaneció vinculado a la producción de conocimiento ( $y$, en especial, a la sistematización de materiales) relacionados con la Historia Económica, desde su responsabilidad institucional en el Departamento de Historia Económica de la Facultad de Ciencias Económicas y de Administración ${ }^{41}$.

Por su parte, Raúl Jacob desarrolló una extensa labor de investigación en aspectos relacionados con el primer proceso industrial, la banca y los sectores hegemónicos de la economía nacional, traducida en numerosas publicaciones ${ }^{42}$, en la reflexión programática sobre las líneas de trabajo a encarar en torno a la industrialización anterior

${ }^{41}$ Cfr.: Benjamín NAHUM, "Reclamación Meillet". Un caso de intervención diplomática francesa en el Uruguay batllista (1912-1914). Montevideo, Ediciones de la Banda Oriental, 1993; Empresas públicas uruguayas. Orígen y gestión. Montevideo, Ediciones de la Banda Oriental, 1993; Deuda externa uruguaya, 1864-1930. Montevideo, Ediciones de la Banda Oriental, 1994; Evolución de la Deuda Externa del Uruguay, 1875-1939. Montevideo, Ediciones de la Banda Oriental, 1995.

42 Cfr.: Raúl JACOB, El Frigorífico Nacional en el mercado de carnes. La crisis de 1928 en el Uruguay. Montevideo, Fundación de Cultura Universitaria, 1979; Breve Historia de la Industria en el Uruguay. Montevideo, Fundación de Cultura Universitaria, 1981; Depresión ganadera y desarrollo fabril. Montevideo, Fundación de Cultura Universitaria, 1981; Crisis y mercado de trabajo: una aproximación a la problemática de los años veinte y treinta. Montevideo, CIEDUR, 1984; Notas sobre el Estado, la Industria y la Banca de Fomento. Montevideo, CIEDUR, 1990; Banca, Estado y Poder Económico. Directorio 1915-1940. Montevideo, CIEDUR, 1991.

R. I., $1997, \mathrm{n}^{\circ} 210$ 
a $1930^{43}$, en la labor docente ${ }^{44}$, y en la constitución de un nuevo ámbito universitario de investigación: el PIHESUC (Programa de Investigación en Historia Económica y Social del Uruguay Contemporáneo, de la Facultad de Ciencias Sociales).

Enfoques monográficos destinados a esclarecer aspectos específicos de los condicionamientos sufridos por el desarrollo económico del país, en particular los referidos a las posibilidades de su financiamiento o a la autonomía de las políticas energéticas, han merecido la atención de investigadores provenientes de la Facultad de $\mathrm{Hu}$ manidades y Ciencias ${ }^{45}$.

En una línea más apegada a los enfoques de Historia Económica previos al '73, tanto los provenientes de los economistas-historiadores (centrados en una Historia de las estructuras económicas y su evolución, más que en el análisis de los hechos económicos o de los contextos socio-culturales de los fenómenos económicos) como de los historiadores marxistas, se ubican las producciones de Julio Millot ${ }^{46}$ (trabajando en el Instituto de Economía, de la Facultad de Ciencias Económicas y Administración), y las del equipo integrado por Lucía Sala de Tourón y Rosita Alonso ${ }^{47}$. Con una finalidad difusora y pedagógica, que si bien no en sus fundamentos teóricos pero sí en sus

43 Cfr.: Raúl JACOB, La industrialización anterior a 1930: Crónica de un debate nunca realizado, en: Martín BUXEDAS - Raúl JACOB, Industria Uruguaya: dos perspectivas. Montevideo, Fundación de Cultura Universitaria-CIEDUR, 1989.

44 Particular relevancia adquirió el Seminario dirigido por Jacob en el marco de las actividades del Departamento de Historia del Uruguay (de la Facultad de Humanidades y Ciencias) que dio lugar a la publicación de: Leonel FERRANDO - Oribe CURES - Miriela LACUESTA - Nelly DA CUNHA - Rossana CAMPODONICO - Isabel EzCURRA, Gremiales empresariales. Unión Industrial Uruguaya. Cámara de Comercio. Cámara Mercantil. Federación Rural. Asociación Rural. Asociación de Consignatarios de Ganado. [Seis fascículos] Montevideo, Facultad de Humanidades y Ciencias de la Educación, 1992.

45 Cfr.: Carlos Zubillaga, El reto financiero. Deuda externa y desarrollo en Uruguay, 1903-1933. Montevideo, Editorial Arca, 1982; Antonio LABRAGA - Mario NUÑEZ Ana M. RODRIGUEZ AyÇAGUER - Esther RUIZ, Energía y política en el Uruguay del siglo $X X$. Montevideo, Ediciones de la Banda Oriental, 1991.

46 Julio Millot - Magdalena Bertino, Historia Económica del Uruguay. Tomo I. Montevideo, Fundación de Cultura Universitaria, 1991.

47 Lucía Sala DE TOURON - Rosita Alonso, El Uruguay comercial, pastoril y caudillesco. Dos Tomos. Montevideo, Ediciones de la Banda Oriental, 1986-1991. 
resultados lo vincula con las producciones precedentes, José Claudio Williman (h) ha iniciado una Historia Económica del Uruguay ${ }^{48}$.

Los aportes menos ortodoxos en el campo de la Historia Económica han correspondido a Luis Bértola ${ }^{49}$ (con un énfasis marcado en las técnicas econométricas) y a Juan Oddone ${ }^{50}$. La obra de este último constituye un excepcional exponente de la renovación historiográfica de los últimos años, al lograr con apoyo en documentación de los National Archives (Washington) y del Public Record Office (Foreign Office, London), un sutil análisis de los cambios y permanencias registrables en la actividad económica como consecuencia del influjo de factores internacionales. La Historia económica se sustrae así a la condena de su incapacidad interpretativa estrechada en el marco nacional.

Uno de los campos de más fértil innovación historiográfica (temática o de enfoque) ha estado reservado a la consideración de los grupos humanos que podrían denominarse marginados o excluídos, en el sentido de ajenos al protagonismo histórico tal como lo concebía la historiografía tradicional (individualista, psicologista y "biografizante"). La Nueva Historia si bien advirtió la dimensión social del acontecer histórico e identificó a estos actores como objeto de su análisis, al priorizar el abordaje cuantitativo en grandes agregaciones y el conocimiento de las estructuras y sus macrotransformaciones, no llegó a profundizar en la condición autónoma que estos sujetos activos adquirieron. Resultó así postergada la apreciación de sus modalidades organizativas, sus tradiciones y mentalidad, su imaginario, sus utopías. A revertir estas carencias vino una producción aún en búsqueda de sus precisos sustentos teóricos. De la mano de tales preocupaciones se asistió al estudio de las mentalidades (¿sensibilidades?), de las mujeres, de los migrantes, de los asalariados, de los enfermos, a través de cauces que comprometían solu-

48 José Claudio Williman (h), Historia Económica del Uruguay, 1811-1900. Montevideo, Editorial Fin de Siglo, 1992; Historia Económica del Uruguay, 1900-1930. Montevideo, Editorial Fin de Siglo, 1994.

49 Cfr.: Luis BERTOLA, La industria manufacturera uruguaya, 1913-1961. Un enfoque sectorial de su crecimiento, fluctuaciones y crisis. Montevideo, Facultad de Ciencias Sociales - CIEDUR, 1991.

50 Juan ODDONE, Uruguay entre la depresión y la guerra, 1929-1945. Montevideo, Fundación de Cultura Universitaria-Facultad de Humanidades y Ciencias, 1990.

R. I., 1997, n. $^{\circ} 210$ 
ciones teóricas, esfuerzos técnicos y opciones metodológicas y discursivas que suponían ciertas rupturas con la producción precedente.

Se trató de una incursión de rango metodológico arduo: ¿dónde estaban las fuentes más pertinentes para "escuchar" las voces de estos protagonistas? ¿cómo superar el carácter iletrado de buena parte de los mismos y, consecuentemente, su ajenidad respecto de la cultura escrita? ¿de qué manera incorporar las referencias a estos sectores realizadas desde el poder (desde cualquier modalidad hegemónica) y desbrozarlas de interferencias ideológicas? ¿cómo desagregar las referencias cuantitativas preexistentes, dando cuenta de las peculiaridades que conformaron la caracterización diferencial de estos sectores, para estimar la gravitación de su número? ¿cómo "dignificar" el testimonio oral, no sólo el originado en la memoria personal sino en la memoria social? ¿qué rango conferir a los vestigios de la cotidianidad en virtud de su preservación discontínua y asistemática? ¿cómo evaluar las múltiples manifestaciones de la intimidad, transmitidas por la tradición o contenidas en la escritura de lo privado? ¿cómo captar los sentimientos, la fe, las convicciones, en su verdadera dimensión interior, más allá de las creaciones que los traducen (y a un mismo tiempo) los distorsionan?

Con una apuesta al análisis de larga duración (tributaria, quizás no totalmente asumida, de la Historia de las mentalidades que fructificara en Francia en el período de entreguerras) la indagación de Barrán sobre la sensibilidad se ubica en el campo de lo imaginario, que al decir de Evelyne Patlagean "está constituído por el conjunto de representaciones que desbordan el límite trazado por los testimonios de la experiencia y los encadenamientos deductivos que éstos autorizan"51. Obra de excepcional repercusión social, esta Historia de la sensibilidad ${ }^{52}$ removió intensamente los objetivos de la preocupación historiográfica, marcando - más allá de algunas de las discutibles tesis que sienta - una inflexión mayor en el desarrollo de la disciplina histórica en el país. Con similar potencialidad polémica, el abordaje de los temas de la enfermedad y la muerte, el cuerpo y la salud, el poder del médico y la sujeción del "paciente", así como las

51 Evelyne PATLAGEAN, La historia de lo imaginario, en: LE GOFF-CHARTIERREVEL (Dir.), La Nueva Historia. Bilbao, Ediciones Mensajero, 1988, p. 302.

52 José Pedro BarRan, Historia de la sensibilidad en el Uruguay. Dos Tomos. Montevideo, Ediciones de la Banda Oriental, 1989-1990.

R. I., $1997, \mathrm{n}^{\circ} 210$ 
creencias, los mitos y los miedos implícitos en una sociedad que se "modernizaba" (pero que dudaba entre los "beneficios del progreso" y las certidumbres de la tradición), han dado a la obra más reciente de José Pedro Barrán ${ }^{53}$ el carácter de un revulsivo a las convenciones historiográficas sostenidas incluso por las dos primeras generaciones de la Nueva Historia.

La historiografía sobre el mundo del trabajo logró sustraerse del servilismo ideológico que pautó su producción hasta el '73; en la década de los ' 80 resultó encarada no como una desagregación del universo social en clave corporativa, sino como un esfuerzo por comprender la complejidad de las relaciones sociales (económicas, culturales, políticas, psicológicas) implicadas por el régimen del salario. El universo a estudio no fue el de las organizaciones sindicales en cuanto instrumentos de reivindicación económica o de lucha social más amplia, sino el del mundo del trabajo (los sectores populares, entendiendo por tales los estratos integrados por "la gente común y humilde" de la sociedad generalmente inserta en una relación asalariada de carácter permanente, aunque también vinculada —en porcentajes apreciables - a los modos de subsistencia propios del "mercado informal"). Este universo comprendió, en las investigaciones producidas ${ }^{54}$, los modos organizacionales de los sectores aludidos (instituciones, estrategias, formas operativas), pero también sus bases demográficas; los avances y retrocesos registrados en sus condiciones de vida; las modalidades del tránsito del artesanado a la industria fabril; las variaciones de los salarios y sus relaciones con el costo de vida; las condiciones de cumplimiento de la relación laboral; la situación de la vivienda, de la alimentación, de la salud y de la educación; las pautas culturales propias (identidades y utopías); el grado de compatibilización posible de los nexos sociales tradicio-

53 José Pedro Barran, Medicina y Sociedad en el Uruguay del Novecientos. Dos Tomos. Montevideo, Ediciones de la Banda Oriental, 1992/1993.

54 Cfr.: Carlos ZubillagA - Jorge BalBIS, Historia del Movimiento Sindical Uruguayo. Cuatro Tomos. Montevido, Ediciones de la Banda Oriental, 1985/1992; Carlos ZUBILLAGA, "Entre luchas y desprecios: los orígenes de la expresión 'carnero"،, en: Cuadernos del CLAEH. $\mathrm{N}^{\circ}$ 45. Montevideo, 1988; Carlos ZuBILlaGA (Comp.), Trabajadores y sindicatos en América Latina. Reflexiones sobre su historia. Montevideo, CLACSO/CLAEH, 1989; Juan RIAL, La alimentación de los sectores populares en Montevido en las primeras décadas del siglo XX. Montevideo, CIESU, 1984; Yamandú GonZALEZ SiERra, 100 Primeros de Mayo en el Uruguay. Montevideo, CIEDUR, 1990.

R. I., 1997, n. $^{\circ} 210$ 
nales con los nuevos roles ocupacionales; la traducción semántica de percepciones que implican el auto-reconocimiento social.

La historiografía sobre la condición social, económica, política y cultural de la mujer irrumpió en los ' 80 -sin perjuicio de reconocer antecedentes en las décadas anteriores, aunque fundamentalmente referidos a un enfoque "sufragista"-, dando lugar a la configuración de un espacio académico propio, que se sumó al de los centros privados de investigación y se integró a la red CLACSO: el GRECMU (Grupo de Estudios sobre la Condición de la Mujer en Uruguay). Si bien el énfasis sobre las dificultades de la mujer en la consolidación de su condición ciudadana ${ }^{55}$ no fue abandonado, la historiografía sobre la mujer buscó otros cauces, relacionándose tanto con la historiografía sobre el movimiento obrero (advirtiendo la importancia de la dimensión laboral femenina $)^{56}$, como con los replanteos metodológicos y las nuevas técnicas de la investigación histórica, en particular, las de la oralidad ${ }^{57}$.

La historiografía sobre la inmigración ha registrado, también, un cambio sustantivo en la década de los '80. De una Historia preocupada por develar el papel del inmigrante como agente decisivo en el proceso de modernización ( $\mathrm{y}$, en consecuencia, orientada a estudiarlo en sus grandes agregaciones), se pasó a una indagatoria cuyo rasgo fundamental estriba en la complejización del cuerpo social, en el análisis de la diversidad. Sobre la base de un cuestionamiento - más o menos explícito- a la tesis que identificó a la sociedad uruguaya como un crisol de razas, con lo que ello comportaba para la configuración de una identidad nacional de signo homogéneo, se ha comenzado a considerar el fenómeno aluvional de las migraciones transo-

\footnotetext{
55 Cfr.: Silvia RodRIguez VIllamil - Graciela SAPRIZA, "Feminismo y política: un análisis del proceso de aprobación del voto femenino en el Uruguay", en: Hoy es Historia. Vol. 1. № 4. Montevideo, 1984; Mujer, Estado y política en el Uruguay del siglo XX. Montevideo, Ediciones de la Banda Oriental, 1984; El voto femenino en el Uruguay ¿conquista o concesión?. Montevideo, GRECMU, 1984.

56 Cfr.: Silvia RodRIGUEZ VIllamil, El trabajo femenino en Montevideo, 1880-1914, en La mujer en el Uruguay. Ayer y Hoy. Montevideo, Ediciones de la Banda OrientalGRECMU, 1983; Graciela SAPRIZA, Obreras y sufragistas ¿Un diálogo imposible?. Montevideo, GRECMU, 1985; Hilamos una historia: la memoria sindical desde las mujeres. Montevideo, FESUR/GRECMU, 1989.

57 Cfr.: Silvia RodrigueZ VILlamIL, Mujeres uruguayas a fines del siglo XIX ¿Cómo hacer su historia?. Montevideo, GRECMU, 1991; Graciela SAPRIZA, Memorias de rebeldía: siete historias de vida. Montevideo, Puntosur/GRECMU, 1988.
} 
ceánicas como fundante de una sociedad de lo diverso. La identidad nacional encuentra así, en la sutil percepción de este fenómeno, las claves de su complejidad, de sus componentes contradictorios, de sus tensiones. De allí los temas que la neohistoriografía sobre la inmigración ha comenzado a priorizar: el asociacionismo étnico, las conductas demográficas de los inmigrantes en relación con el modelo demográfico de la modernización en el país, las relaciones de los colectivos de inmigrantes con la emergencia del movimiento sindical uruguayo, la incidencia de la inmigración europea en la configuración del empresariado local, las prácticas cultuales de los inmigrantes en vinculación con la religiosidad popular en Uruguay ${ }^{58}$.

\section{CODA}

Los signos más reveladores de los cambios producidos en la historiografía uruguaya a partir de la experiencia de las dos últimas décadas, quizás deban ubicarse tanto en el plano teórico-metodológico como en el técnico y en el institucional.

En el terreno teórico-metodológico se destaca la ampliación cronológica del objeto de estudio, que ha venido a marcar una diferencia sensible respecto de la preocupación historiográfica de la primera generación de la Nueva Historia. De la concentración en el proceso de modernización (desde la emergencia del proyecto de transformación agropecuaria hacia 1870 , hasta los efectos locales de la crisis

$58 \mathrm{Cfr}$. al respecto los diversos trabajos que componen el volumen colectivo Fernando J. Devoto et al., L'emigrazione italiana e la formazione dell'Uruguay moderno. Torino, Edizioni della Fondazione Giovanni Agnelli, 1993: María Magdalena CAMOU - Adela PELleGrino, Dimensioni e caratteri demografici dell'emigrazione italiana in Uruguay, 1860-1920; Juan Antonio ODDONE, La politica e le immagini dell'immigrazione italiana in Uruguay, 1830-1930; Carlos ZUBILlAGA, Religiosità, devozione popolare e immigrazione italiana in Uruguay; Alcides BERETTA CURI, Il contributo dell'emigrazione italiana allo sviluppo economico dell'Uruguay, 1875-1918; Oscar MOURAT, Gli italiani e l'acquisizione della proprietà nell'Uruguay moderno; Jorge MORENO-Susana ANTOLA-Mary GALBIATTIElena MAZZINI-Cecilia PONTE, L'apporto italiana all'immagine urbana di Montevideo nell'edilizia civile. Asimismo: Carlos ZuBILlaga, Hacer la América. Estudios históricos sobre la inmigración española al Uruguay. Montevideo, Editorial Fin de Siglo, 1993; Alcides BERETTA CURI, Pablo Varzi. Un temprano espíritu de empresa. En torno a los orígenes de la industrización uruguaya, 1875/1930. Montevideo, Editorial Fin de Siglo, 1993.

R. I., 1997, n. $^{\circ} 210$ 
de 1929) el interés de los historiadores se ha expandido hacia el pasado $^{59}$ y hacia el presente ${ }^{60}$.

También debe contabilizarse en el plano teórico-metodológico el cambio observable respecto a la diversificación de los sujetos, respondiendo a un interés por indagar la pluralidad de roles concurrente en la configuración de una sociedad aluvional. Sin ceñir las opciones metodológicas a la alternativa cerrada individuo/sociedad, se ha recuperado la significación de las organizaciones intermedias de la más variada naturaleza (gremiales, étnicas, educacionales, corporativas, de género, religiosas), renovando el valor de la biografía e incursionando en técnicas prosopográficas.

Quizás el rasgo más renovador en la perspectiva teórica haya que referirlo a la autopercepción del colectivo de historiadores como partícipes de un campo crecientemente profesionalizado. Las prácticas laborales asumidas a través de los centros privados de investigación durante el período dictatorial, la incorporación al ámbito universitario de criterios de evaluación frecuentemente sustentados en la competitividad, la subsistencia de la apelación al financiamiento de las investigaciones mediante modalidades concursables, la demanda sostenida de producción ajustada al interés del lector/consumidor por parte de la industria editorial, la vinculación de algunos integrantes de la academia con los medios masivos de comunicación (en especial, radio y televisión) y sus condicionamientos promocionales, han ido modificando el perfil del historiador, al

\footnotetext{
59 De este modo ha comenzado a recuperarse la preocupación por la Historia Colonial (espacio de interés privilegiado de la historiografía tradicional), a la luz de interrogaciones relevantes y con sustento en rigurosa exhumación de fuentes de archivos españoles y americanos. Arturo Ariel BENTANCUR es el más calificado exponente de esta vertiente; cfr. de su producción: Contrabando y contrabandistas. Historias coloniales. Montevideo, Editorial Arca, 1982; Don Cipriano de Melo. Señor de fronteras. Montevideo, Editorial Arca, 1985; Francisco de Medina. La empresa de la discordia. Montevideo, Editorial Arca, 1987; La primera burocracia montevideana (1724-1814), en: Ediciones del Quinto Centenario. 2: América Latina y España: de la Colonia a la constitución de los Estados Nacionales. Montevideo, Universidad de la República, 1992.

60 El límite hacia el presente más aceptado por la historiografía uruguaya de los años ' 60 y '70 se ubicó en el primer lustro de la década de 1930, que se correspondía tanto con el fenómeno político de la ruptura del orden institucional (el golpe de Estado de 1933) como con la crisis socio-económica que reflejaba el cimbronazo del sistema capitalista. En la última década y media la historiografía incursionó frecuentemente en el acontecer más reciente, centrando buena parte de su preocupación en el proceso de emergencia de la dictadura militar, su consolidación y su agotamiento. No ha sido ajeno a este aggiornamento el incremento del diálogo entre historiadores y politólogos.
} 
tiempo que instaurado una discusión sobre los límites de la profesionalización y la necesidad de preservar un equilibrio entre ésta y cierta "militancia" intelectual considerada como ingrediente inexcusable de la vocación.

Respecto al plano técnico, resulta ineludible advertir la superación de la desconfianza ante la oralidad, que en los '60 pautaba todavía la formación universitaria en la disciplina. Una incipiente reflexión teórica sobre la virtualidad de las técnicas de oralidad y sus aplicaciones al conocimiento histórico ${ }^{61}$, así como el desarrollo de programas específicos de investigación en Historia Oral ${ }^{62}$ y la inclusión de las técnicas correspondientes en el curriculum universitario $^{63}$, constituyen los ejemplos de una opción metodológica que ha vigorizado ciertos emprendimientos investigativos en Historia contemporánea y facilitado, por otra parte, el diálogo interdisciplinario.

Asimismo constituye expresión de la renovación técnica la ampliación del espectro heurístico, que ha permitido, a través de la tipificación de nuevas categorías de fuentes (con los más variados soportes) y de los aportes interpretativos que para considerarlas provienen de variados campos disciplinarios (ciencias de la comunicación, sociolingüística, psicoanálisis, semiología), enriquecer la noción de hecho histórico.

En cuanto al plano institucional, la sobrevivencia de estructuras esclerosadas (el Instituto Histórico y Geográfico del Uruguay, el Museo Histórico Nacional) ha dejado nuevamente a la Universidad de la República como el espacio académico hegemónico, con la novedad de la multiplicación en el seno de la misma de los ámbitos

61 Cfr.: Carlos ZuBillaga, "Historia Oral: la voz de los protagonistas" en: Cuadernos del CLAEH. $\mathrm{N}^{\circ}$ 36. Montevideo, Octubre-Diciembre 1985; Laura BERMUDEZ-María CAMOU-Dieter SCHONEBOHM, Las Historias de Vida. Una vieja nueva metodología. Montevideo, GRECMU, 1991.

62 Entre 1987 y 1988 Carlos Zubillaga dirigió en el CLAEH el proyecto de Historia Oral: Archivo de la Memoria Popular, con el auspicio del Consejo Nacional de Investigaciones Científicas y Técnicas (CONICYT) y el Fondo de Fomento de las Artes (UNESCO).

Desde 1986, asimismo, se viene desarrollando en el Departamento de Historiología de la Facultad de Humanidades, el proyecto La voz de los historiadores, que a partir de 1993 cuenta con el respaldo de la Comisión Sectorial de Investigación Científica (CSIC) en el marco del PRIOLE (Programa Interdisciplinario de Oralidad y Lenguas Extranjeras); en esta etapa ha participado activamente en el proyecto Laura Reali.

63 En el curso de Técnicas de la Investigación Histórica, de la Licenciatura de Historia de la Facultad de Humanidades, el profesor Dante Turcatti desarrolla un Seminario permanente sobre Influencia del trabajo en la familia: Montevideo, 1940/1950. 
docentes o de investigación abocados al cultivo de la disciplina. En octubre de 1992 tuvo lugar el Primer Encuentro Universitario de Ciencias Históricas, cumplido en la Facultad de Humanidades y Ciencias de la Educación, que se constituyó en una fecunda instancia de diálogo e intercambio ${ }^{64}$ entre los docentes e investigadores universitarios en Historia ${ }^{65}$, con vistas a la superación de las diferencias básicamente operativas que por lo general limitan (en instituciones de estas características) el desarrollo profesional y la economía de esfuerzos en la acumulación del conocimiento.

The author provides an overview of the historical research done in Uruguay in the period from the eve of the military coup of June, 1973 to the present a period of the systematic renewal in this area of scholarship. Over these 25 years there have been significant changes in theoretical criteria, research interests, patterns of cooperation among historians, diffusions of their work, and professionalism in the field.

\footnotetext{
${ }^{64}$ Los objetivos del Encuentro fueron: 1- relevar el volumen y la significación académica de los ámbitos universitarios en los que se cultiva el conocimiento histórico; 2estudiar modalidades eventuales de coordinación, complementación o distribución de acciones docentes, de investigación y de extensión entre los ámbitos identificados; 3- proponer acciones de fortalecimiento de la disciplina, sobre la base de la mutua prestación de servicios que potencialicen idoneidades específicas; 4- establecer un directorio de investigaciones en ciencias históricas de la Universidad en su conjunto; 5- reclamar para la Universidad de la República una participación más activa en el asesoramiento a instituciones públicas en materias que involucren a las Ciencias Históricas; 6- encarar acciones editoriales conjuntas; 7- propender al reconocimiento social y, eventualmente, jurídico de la profesión de historiador, tendiendo asimismo a la determinación de criterios deontológicos en el ejercicio de la misma.

65 Participaron docentes de las Facultades de Arquitectura, Derecho, Ciencias Económicas y Administración, Ciencias Sociales, Humanidades y Ciencias de la Educación, Medicina, y Psicología, y de las Escuelas de Bibliotecología y Ciencias Afines, Universitaria de Música, y Nacional de Bellas Artes.
} 